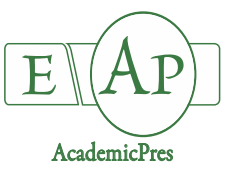

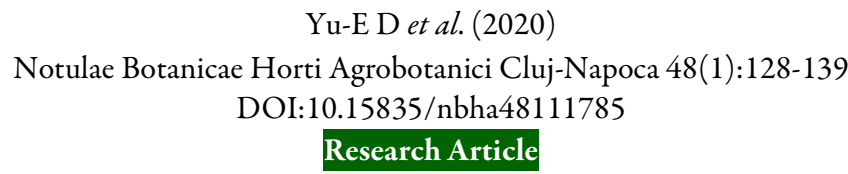

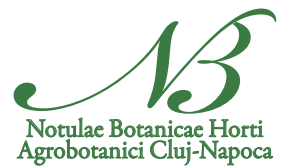

\title{
Identification and characterization of a circadian clock-associated pseudo-response regulator 7 gene from trifoliate orange
}

\author{
Yu-E DING ${ }^{1 a}$, Wenkai HUANG ${ }^{2 b}$, Bo SHU ${ }^{1}$, Ying-Ning ZOU ${ }^{1}$, \\ Qiang-Sheng WU ${ }^{1,3 *}$, Kamil KUČA ${ }^{3}$ \\ ${ }^{1}$ Yangtze University, College of Horticulture and Gardening, Jingzhou, Hubei 434025, \\ China;1908417953@qq.com; 786212719@qq.com; zouyingning@163.com;wuqiangsh@163.com(*correspondingauthor) \\ ${ }^{2}$ Huazhong Agricultural University, College of Life Science and Technology, Wuban 430070, China; huangwk@webmail.hzau.edu.cn \\ ${ }^{3}$ University of Hradec Kralove, Faculty of Science, Department of Chemistry, Hradec Kralove 50003, Czech Republic; kamil.kuca@ubk.cz \\ ${ }^{a, b}$ These authors contributed equally to the work
}

\begin{abstract}
Circadian clock is usually involved in many physiological processes of plants, including responses to abiotic stress, whilst pseudo-response regulator 7 (PRR7) gene is the main component of the circadian clock. In this study, the cDNA of the PRR7 gene was obtained from trifoliate orange (Poncirus trifoliata). Based on the sequence analysis, the PtPRR7 gene had an open reading frame of $2343 \mathrm{bp}$, encoded 780 amino acids, and contained proteins of the REC and CCT domains. Subcellular localization indicated that PtPRR7 was mainly localized in the nucleus and a small amount of cytoplasm. qRT-PCR analysis revealed the highest expression level of PtPRR 7 in roots than in both shoots and leaves. The PtPRR7 gene during 24 hours of soil water deficit exhibited a circadian rhythmic expression pattern: the expression peak at 9:00 am in leaves and at 21:00 pm in roots. Drought treatment affected PtPRR7 gene expression. Such data provide important references for understanding the characteristics of PtPRR7 gene in citrus plants.
\end{abstract}

Keywords: citrus; diurnal rhythm; drought; pseudo-response regulator

\section{Introduction}

Due to the rotation of the earth, there are daily changes in the surrounding environment of plants, mainly including light and temperature (Bhattacharya et al., 2017). To adapt to this environment, plants must respond with a circadian clock (Nohales and Kay, 2016). Meanwhile, the circadian clock regulates the synthesis and utilization of resources in a transient manner, and ultimately enhances the plant's adaptability and resistance to stress (Seo and Mas, 2015). Circadian clock usually controls many important physiological processes of plants, such as hypocotyl elongation, leaf movement, stomatal switching, flowering, etc. (Greenham and McClung, 2015). It can enable organisms to anticipate fluctuations in the external environment, coordinate vital physiological activities and developmental processes at appropriate times of the day, and improve adaptability to the environment (Yerushalmi and Green, 2009). 
In plants, the transcription can be regulated by the circadian clock (Covington et al., 2008; Michael et al., 2008). Liu et al. (2013) observed that the principal component of the circadian clock in Arabidopsis was pseudo-response regulator 7 (PRR7) directly involved in the inhibition of plant growth, light signals and stress responses. Moreover, the PRR7 expressions are conserved among different plant species (Khan et al., 2010; Filichkin et al., 2011). Components of circadian clock interacted to establish a complex network of feedback loops at the transcription level (De Caluwé et al., 2016). Two homologous MYB transcription factors, circadian clock associated 1 (CCA1) and late elongated hypocotyl (LHY), peaked near dawn to activate PRR9 and PRR7 expressions (Farré et al., 2005). PRR5, PRR7 and PRR9 protein levels peak and end a day, thereby inhibiting CCAI and LHY expressions (Farré and Kay, 2007; Nakamichi et al., 2010). The mutant having PRR5, PRR7, and PRR9 was more resistant to drought and cold, with the increased abscisic acid (ABA) levels (Fukushima et al., 2009; Syed et al., 2015). As a result, PRR-related genes are strongly correlated with drought tolerance (Juliana et al., 2014; Li et al., 2017).

Citrus is one of the most widely cultivated fruit crops (Wu et al., 2013,2019), which often suffers from soil water deficit during summer and autumn, thereby, causing poor vigor growth and low fruit quality (Poggi et al., 2007; Osakabe et al., 2013; Santana-Vieira et al. 2016; He et al., 2020). Trifoliate orange (Poncirus trifoliata L. Raf.) is an important rootstock of citrus plants used in Southeast Asia and also extreme droughtsensitive plant (Zhang et al., 2020). Studies on circadian clock genes are focused on model plants such as Arabidopsis thaliana and rice (Garg et al., 2019; Jain et al., 2018), while the information regarding the PRR7 gene in trifoliate orange is scarce. The objective of the present work is to clone the $P R R 7$ gene from trifoliate orange and also analyze the characteristics and the functions of the gene in response to drought stress.

\section{Materials and Methods}

\section{Cloning and sequence analysis}

Leaves of trifoliate orange were ground with liquid nitrogen. Total RNA was extracted using the kit PrimeScriptTM RT Master Mix (Baori Medical Biotechnology Beijing Co., Ltd., China), and reversely transcribed into cDNA using the kit PrimeScriptTM RT reagent Kit with gDNA Eraser (Baori Medical Biotechnology Beijing Co., Ltd., China). Specific primers for PCR amplification were developed according to the sweet orange database (http://citrus.hzau.edu.cn/orange/index.php) (Table 1). The RT-PCR products were purified and connection to the 1300-35S-YFP vector. The ligation product was transformed into Escherichia coli, and the positive clones were chosen for sequencing. Sequence alignment was carried out using the DNAMAN (version 5.2.2) program. A phylogenetic tree was constructed using MEGA-X for the amino acid sequences of the circadian clock gene from different plants. The phylogenetic tree generation algorithm was obtained by the Neighbor-joining (NJ) method (Saitou and Nei, 1987). We used the bootstrap to verify the phylogenetic tree and define the number of repetitions to 1000 (Sitnikova et al., 1995). Structural prediction of the gene was calculated as per the SWISS-MODEL online tool (bttps://swissmodel.expasy.org/).

\section{Subcellular localizations of PtPRR7}

The subcellular localization vector was designed to construct primers PtPRR7-F/R (Table 1) and a target band was used as a template to amplify the target band. The recombinant method was inserted into the EcoRI / SpeI site of the 1300-35S-YFP vector to construct a satisfactory subcellular localization vector (PtriPRR7RRFP) to transform Agrobacterium EHA105. The transient expression method was utilized to inject Agrobacterium that successfully transformed the target plasmid into tobacco leaves, and cultured for 48 hours in the dark. Using the 3302Y3 empty vector as a control, the laser confocal microscope (Leica SP-5, Leica, Wetzlar, Germany) was used to observe the distribution of YFP fluorescence in the cells. 
Table 1. Genes-specific primer sequences used in this study

\begin{tabular}{|c|c|c|}
\hline Primer names & Primer sequence $\left(5^{\prime} \rightarrow 3^{\prime}\right)$ & Purpose \\
\hline \multirow{2}{*}{ PtPRR7 } & F: aagagacaggatccgaattcatggACGTTGATGGCAAAGCAGAC & Vector construction of Subcellular \\
\cline { 2 - 2 } & R: acctccgaccggtgcactagtAGAACATTCTGGTTCCCTGC & localization \\
\hline \multirow{2}{*}{ PtPRR7 } & F: TAGGAGCACACAAGAGCAGC & \multirow{2}{*}{ qRT-PCR and qPCR } \\
\cline { 2 - 2 } & R: TTGTGGAACAGCTTCAGCCA & \multirow{2}{*}{ Reference gene for qRT-PCR } \\
\hline \multirow{2}{*}{$\beta$-Actin } & F: CCGACCGTATGAGCAAGGAAA & \\
\cline { 2 - 3 } & R: TTCCTGTGGACAATGGATGGA & \\
\hline
\end{tabular}

\section{Plant cultures and drought treatments}

Seeds of trifoliate orange were surface-sterilized with $70 \%$ alcohol solutions for 10 minutes, washed five times with distilled water, and germinated in sterilized $\left(121^{\circ} \mathrm{C}, 0.1 \mathrm{MPa}, 1 \mathrm{~h}\right)$ river sand in a light incubator. The environmental conditions were set to a temperature of $28^{\circ} \mathrm{C} / 20^{\circ} \mathrm{C}(16 \mathrm{~h}$ day $/ 8 \mathrm{~h}$ night $)$, a light intensity of 1200 Lux, and a constant relative humidity of $68 \%$. Three trifoliate orange seedlings having five leaves were transplanted into a plastic pot supplied with autoclaved substrate growth substrate (soil:sand $=5: 1 \mathrm{v} / \mathrm{v}$ ). These plants were grown in a greenhouse without any temperature control equipment. The soil water of the pots was regularly maintained in $75 \%$ of the maximum soil water holding capacity for 11 weeks. Subsequently, 18 pots were kept under $75 \%$ of the maximum soil water holding capacity conditions, while the other 18 pots were subjected to $55 \%$ of the maximum soil water holding capacity for 11 weeks. Soil moisture was controlled by daily weighing with an electronic scale. In order to analyze the response pattern of $P t P R R 7$ to drought stress, nine seedlings from three pots were kept in the target soil water content and then harvested every four hours from 9:00 am of the first day to 5:00 am of the next day, and a total of six harvests were done.

\section{qRT-PCR analyses}

Total RNA of leaves and roots was extracted and the cDNA was synthesized as indicated in 2.1 Cloning and sequence analysis. qRT-PCR analysis was performed using the ChamQ ${ }^{\mathrm{mw}}$ Universal SYBR qPCR Master Mix Kit (Vazyme Biotech Co., Ltd). The qRT-PCR primers were developed based on the sequences obtained (Table 1). The qRT-PCR reaction was previously described by He et al. (2019). CFX96 real-time PCR detection system (BioRad Laboratories, USA) was used. Each sample had three biological replicates with three technical replications. The relative quantification was calculated using the $2^{-\Delta \Delta C T}$ method (Livak and Schmittgen, 2001), and $\beta$-actin was used as a reference gene to normalize gene expression.

\section{Results}

\section{Acquisition of PtPRR7 full-length and bioinformatical analysis}

The PtPRR7 gene (GenBank accession No: MN890019) had an open reading frame of 2343 bp and encodes 780 amino acids. Its molecular weight is $84845.39 \mathrm{kDa}$ and its isoelectric point is 7.54 . The protein instability index was 49.28 , and the average hydrophobicity was -0.731 . The first three amino acid contents were alanine (31.7\%), glycine (25.7\%) and threonine (23.4\%), and the fat index was 71.50 . At the same time, secondary structure predictions revealed that approximately $62.31 \%$ of the PtPRR7 protein sequence had random coils and $9.87 \%$ extended strands, and the $\alpha$-helix and $\beta$-turn were $25.38 \%$ and $2.44 \%$, respectively (Figure 1). The NetPhos (http://www.cbs.dtu.dk/services/) predicted 52 serine, 10 threonine and 5 tyrosine in the PtPRR7 protein as potential phosphorylation sites (Figure 2a). Molecular simulation indicated that the predicted three-dimensional structure of the PtPRR7 protein consisted of a two-component system response regulator and a GDGEF domain protein (Figure $2 b$ ). 


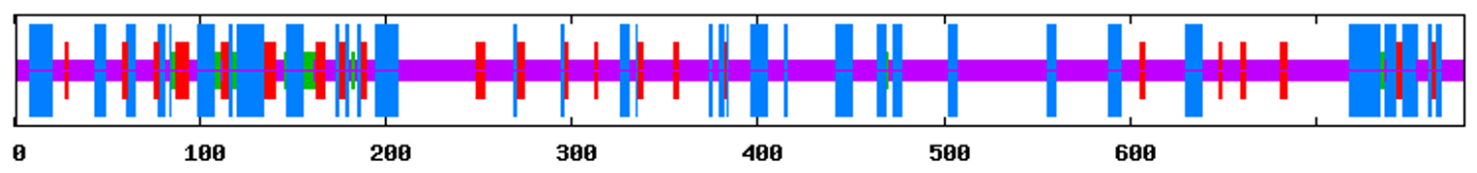

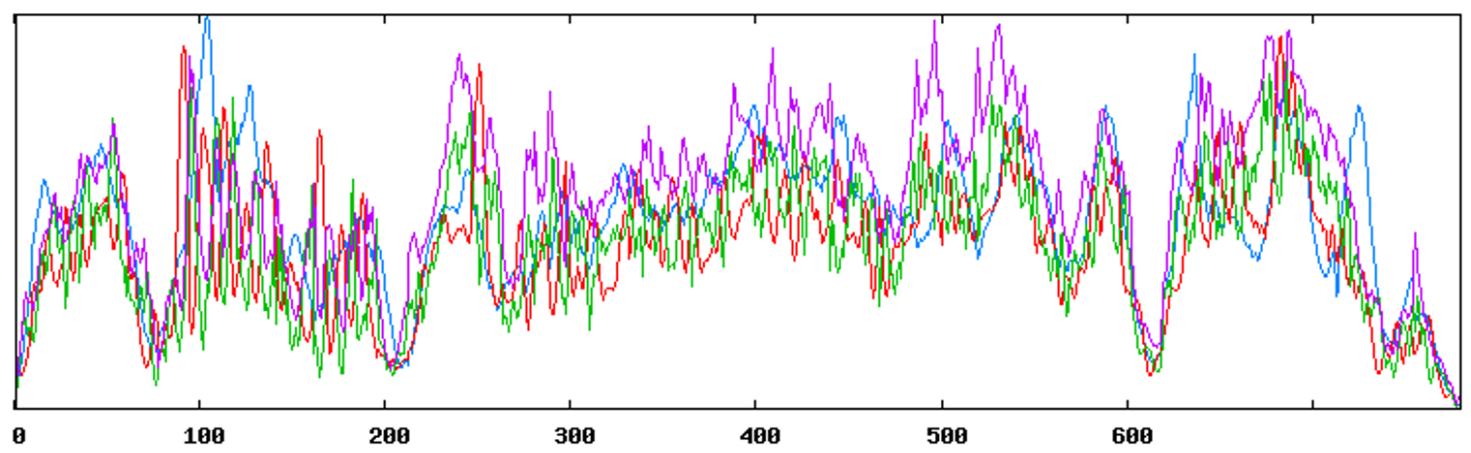

Figure 1. Distribution of the secondary structure of the PtPRR7 gene. The vertical lines in the picture above are $\alpha$-helix (blue), extended strand (red), $\beta$-sheet (green), and random curl (purple)

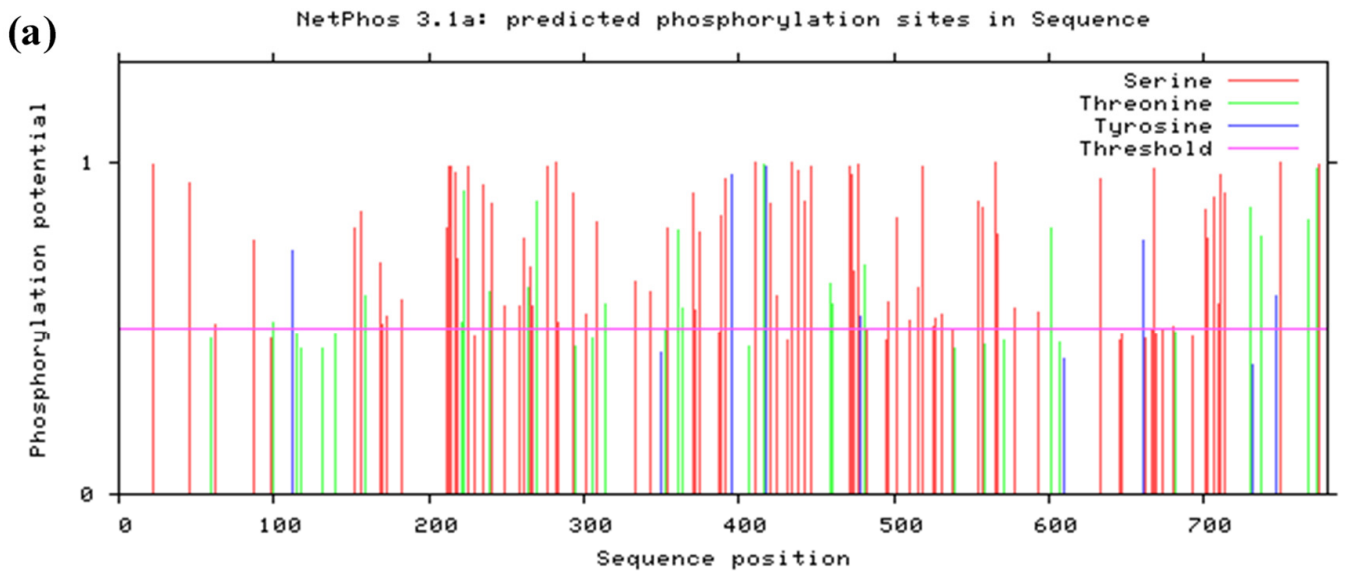

(b)

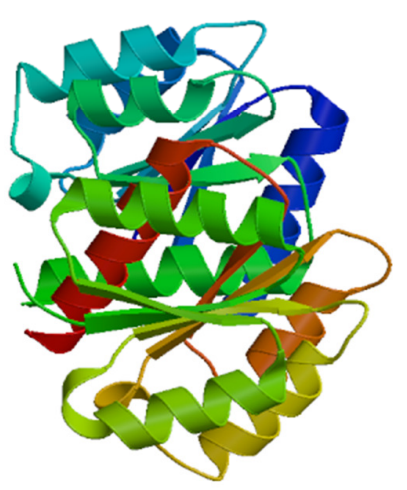

Figure 2. Analysis of amino acid sequences (a) and three-dimensional structure (b) of PtPRR7 protein 
The amino acid sequence of the PtPRR7 protein was compared with the amino acid sequence of PRR7 protein of nine additional plant species by DNAMAN. The results showed that the sequence homology of PRR7-type proteins from different plant families was 75.54\% (Figure 3), indicating that the PRR7-type protein has high sequence conservation.

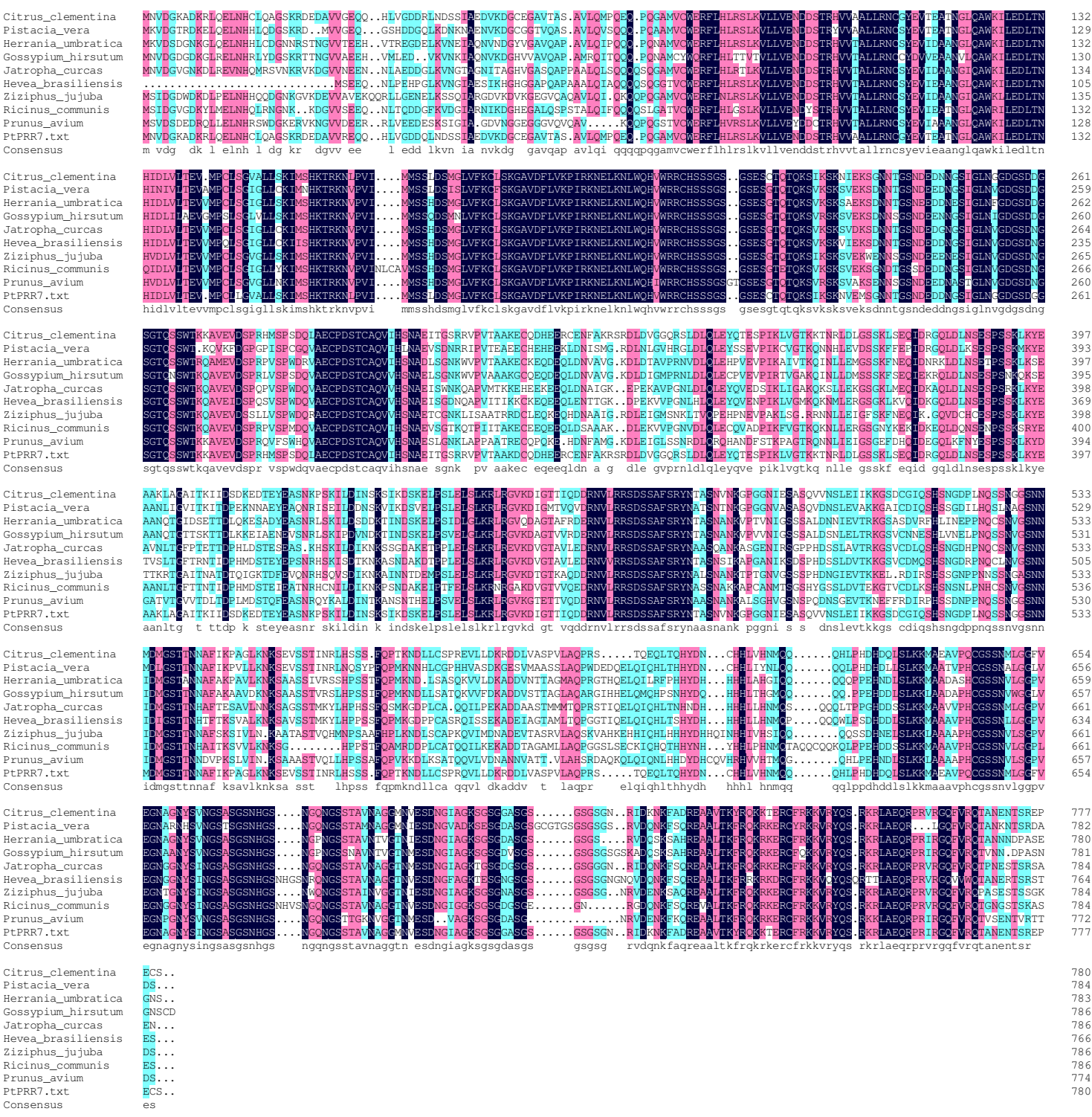

Figure 3. Multiple alignments of the amino acid sequence of PtPRR7 with other PRR7 family proteins. Conserved amino acid residues in all proteins are shaded in black. Red shading indicates a similarity of $75 \%$ or more, and blue shading indicates an acquaintance of $50 \%$ or more 
The phylogenetic relationship between PRR7 and its homologues indicated that all proteins had different clusters. PtPRR7 is grouped with the member of Citrus clementina, and is closest to the PRR7 of Pistacia vera (Figure 4). And the branch of the phylogenetic tree showed that the PRR proteins of the dicotyledonous and monocotyledonous plants were branched into two groups.

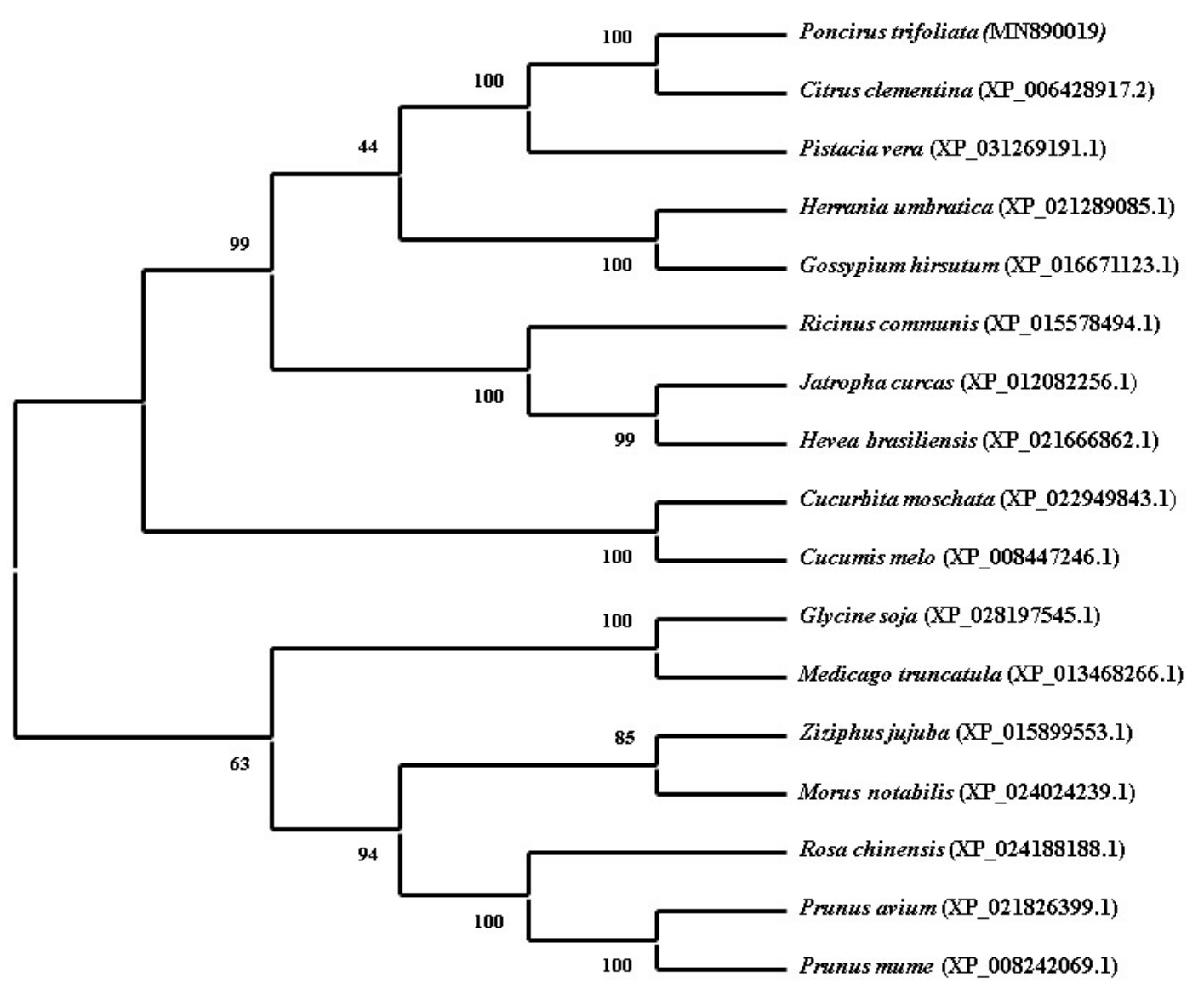

Figure 4. Phylogenetic relationship between PPRR7 constructed with MEGA-X software and the homologs of other plants

\section{Subcellular localizations of PtPRR 7 gene}

The results of subcellular localization showed that PtPRR7 was localized in the nucleus. In order to analyze the subcellular localization of the PtPRR7 protein, a 35 S:PtPRR7:YFP vector was constructed, with $35 S$ :: YFP empty as a control. After the plasmid was successfully transformed into Agrobacterium, the leaves of Bunsen tobacco were injected. After 48 hours of dark culture, the yellow fluorescent protein distribution was observed under Leica SP-5 laser confocal microscopy. As shown in Figure 5, it was found that the PtPRR7 gene is mainly located in the nucleus, and there is a weak signal on the plasma membrane. Combined with the prediction results, PtPRR7 has a nuclear localization signal and homologous genes can function as transcription factors. It is speculated that PtPRR7 is also located in the nucleus as a transcription factor and plays a role in transcription regulation. 


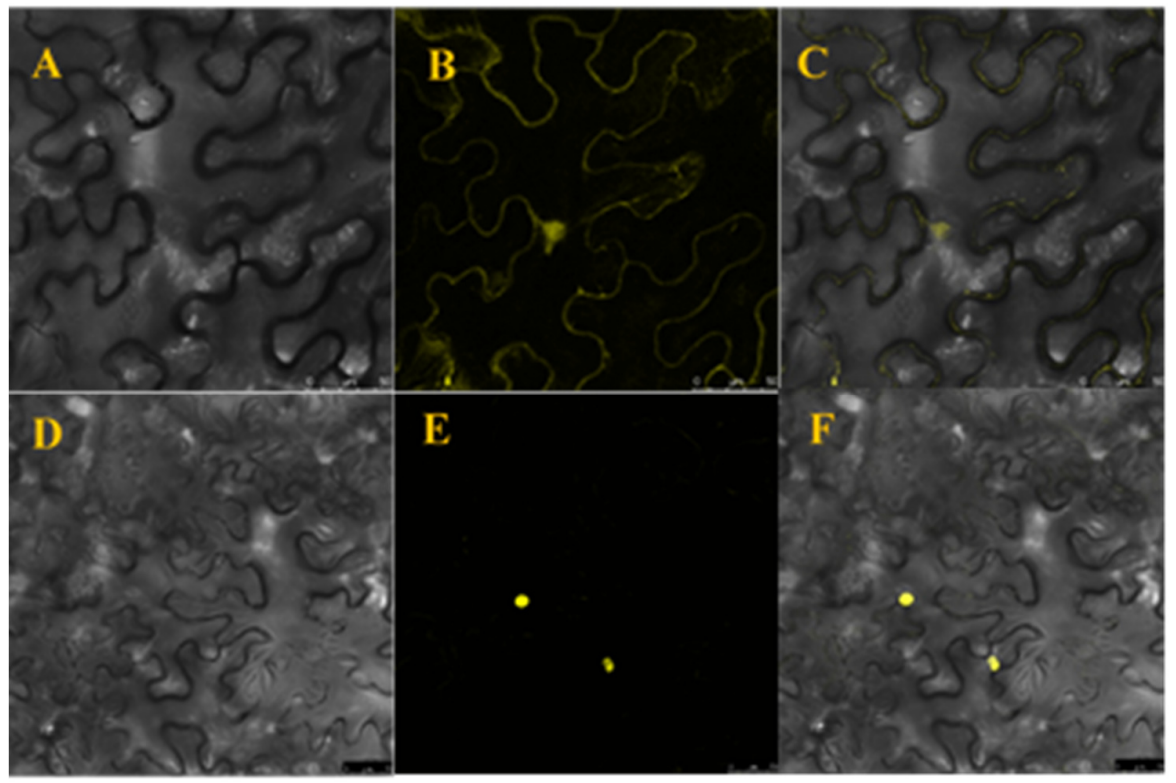

Figure 5. Subcellular localization of PtPRR7. A-C: the positive control of 35S:: YFP. D-F: 35 S :: PtPRR7: YFP fusion protein result. A and D: bright field; B and E: YFP; C and F: merged

\section{Tissue-specific expression patterns of PtPRR 7 gene}

The tissue expression profile of PtPRR7 was detected using qRT-PCR. The results showed that PtPRR7 could be expressed in roots, stems and leaves (Figure 6). The expression level of PtPRR7 in roots was significantly1.7-times and 2.3-time higher than that in stems and leaves, respectively.

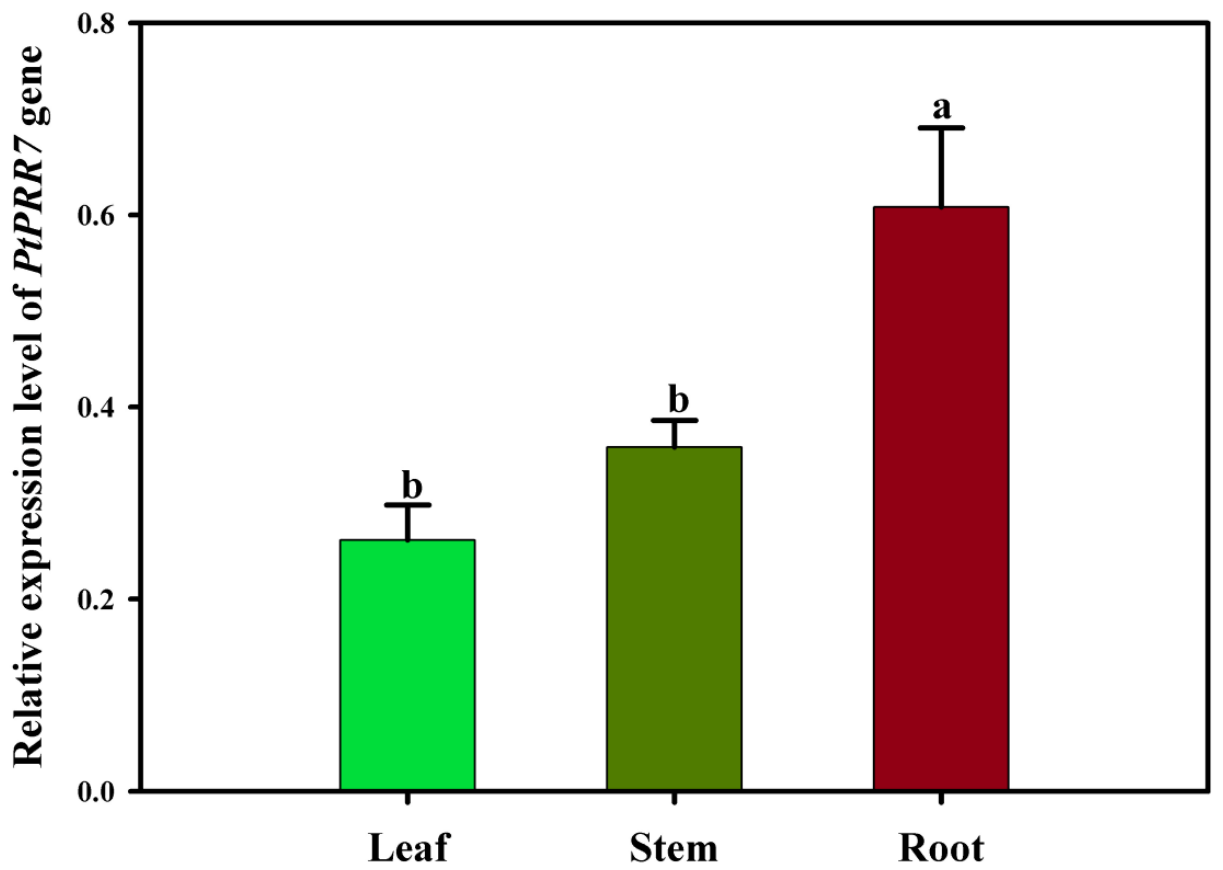

Figure 6. The tissue-specific expression of PtPRR7 gene. Data (means $\pm \mathrm{SD}, n=3$ ) are significantly different $(P<0.05)$ if followed by different letters above the bars 
Expression patterns of PtPRR7 in response to drought stress

Time-specific expression pattern of PtPRR7 gene showed strong periodicity, with obvious peaks and troughs (Figure 7). During 24 hours of soil water deficit between 9:00 am of the first day to 5:00 am of the next day, the expression of PtPRR7 gene in leaves reached its peak at 9:00 am of the first day. The expression level of PtPRR7 gene in root reached its peak at $21: 00 \mathrm{pm}$ of the first day. Compared with well-watered treatment, drought stress treatment induced an up-regulated expression of PtPRR7 in leaves at 9:00 am of the first day and a down-regulated expression of PtPRR7 in leaves at 21:00 pm of the first day, 1:00 am of the next day, and $5: 00 \mathrm{pm}$ of the next day (Figure 7a). Additionally, in roots, drought treatment induced the expression of PtPRR7 at 13:00 am and 21: am of the first day while down-regulated the expression of PtPRR7 at 1:00 am and 5:00 am of the next day (Figure 7b). At the same time, the expression of drought treatment at other time points was lower than that of normal water. During 24 hours of drought stress, the expression level of PtPRR7 gene in leaves and roots was the lowest at 1:00 am of the next day.

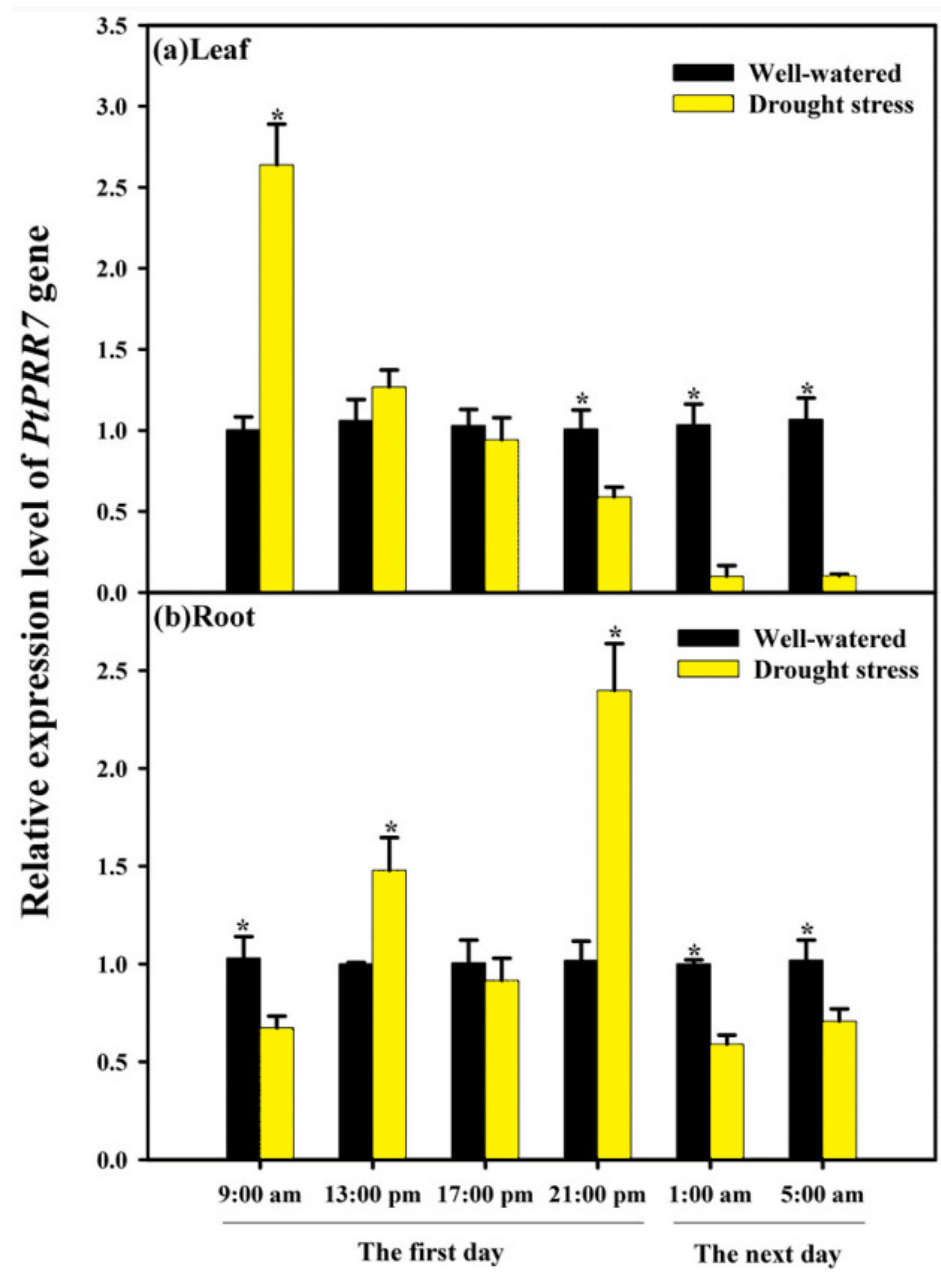

Figure 7. Time-specific expression patterns of PtPRR7 gene in leaf (a) and root (b) in response to wellwatered and drought stress conditions. Data (means \pm SD, $n=3$ ) are significantly different $(P<0.05)$ if followed by asterisks $\left({ }^{*}\right)$ above the bars 


\section{Discussion}

In this study, the PRR7 gene was firstly cloned in trifoliate orange. The comparison of sequencing showed that the PRR7 gene had conserved REC and CCT domains at the N-or C-terminus of the amino acid sequences of the PRR genes in other plants. This is in agreement with other plants (Makino et al., 2000; Farré and Liu, 2013). The phylogenetic tree showed that the PRR protein of dicotyledonous and monocotyledonous plants formed two groups that are far. PtPRR7 is closest to PRR7 of Citrus clementina. Obviously, the PRR gene of citrus is evolutionarily conserved as a congeneric gene (Murakami et al., 2007; Takata et al., 2010).

Previous studies indicated that acidic isomers of PRR proteins in tobacco, such as PRR-1a, PRR-1b, and PRR-1c, existed in extracellular space (Dixon et al., 1991; Elvira et al., 2008; Riviere et al., 2008). Our results showed that PtPRR7 is a slightly acidic protein with an isoelectric point of 7.54. On the other hand, it is consistent that PtPRR7-YFP has a small amount of fusion outside the cell. Some literatures indicate that PRR is involved in the regulation of transcription factors and transcription inhibitors (Para et al., 2007), and most transcription factors are also localized in the nucleus. Subcellular localization results showed that the protein expressed by PtPRR7 was mainly localized in the nucleus, which was consistent with the expected results. qRTPCR analysis revealed that the PtPRR7 gene was expressed in roots, stems, and leaves, whilst the PtPRR7 gene was the highest expression in roots. The results are in agreement with a study in mulberry (Fang et al., 2019).

Time-expression analysis showed that the PtPRR7 gene had the circadian feature in leaves and roots of trifoliate orange during drought stress, which agrees with earlier studies in Arabidopsis and turnip (Kim et al., 2007). Our results also indicated that the expression of PtPRR7 gene in leaves during drought stress peaks at 9:00 am, because PRRs family genes are expressed in the morning (Wang et al., 2013). Interestingly, the expression of PtPRR7 gene in roots peaked at 21:00 pm. In Arabidopsis, the sensitivity of PRRs was linked to light (Farré and Liu, 2013). PRR7 gene regulated the upstream regions of several genes involved in ABA signaling pathways, a mechanism of drought tolerance (Chen et al., 2008; Du et al., 2012; Wang et al., 2018). Therefore, the PtPRR7 gene may improve drought tolerance by regulating genes implicated in ABA signaling pathways, which have yet to be studied. In addition, it has been noted that the expression of PRR genes was induced by other abiotic stress, besides drought (Atamian and Harmer, 2016; Nakamichi et al., 2016).

\section{Acknowledgements}

This study was supported by the National Key Research and Development Program of China (2018YFD1000300) and the Plan in Scientific and Technological Innovation Team of Outstanding Young Scientist, Hubei Provincial Department of Education (T201604).

\section{Conflict of Interests}

The authors declare that there are no conflicts of interest related to this article.

\section{References}

Atamian HS, Harmer SL (2016). Circadian regulation of hormone signaling and plant physiology. Plant Molecular Biology 91:691-702.

Bhattacharya A, Khanale V, Char B (2017). Plant circadian rhythm in stress signaling. Indian Journal of Plant Physiology 22:147-155. 
Chen H, Zhang J, Neff MM, Hong SW, Zhang H, Deng XW, Xiong L (2008). Integration of light and abscisic acid signaling during seed germination and early seedling development. Proceedings of the National Academy of Sciences 105:44954500.

Covington MF, Maloof JN, Straume M, Kay SA, Harmer SL (2008). Global transcriptome analysis reveals circadian regulation of key pathways in plant growth and development. Genome Biology 9:R130.

De Caluwé J, Xiao Q, Hermans C, Verbruggen N, Leloup JC, Gonze D (2016). A compact model for the complex plant circadian clock. Frontiers in Plant Science 7:74.

Dixon DC, Cutt JR, Klessig DF (1991). Differetial targeting of the tobacco PR-1 pathogenesis-related proteins to the extracellular space and vacuoles of crystal idioblasts. The EMBO Journal 10:1317-1324.

Du SY, Zhang XF, Lu Z, Xin Q, Wu Z, Jiang T, Lu Y, Wang XF, Zhang DP (2012). Roles of the different components of magnesium chelatase in abscisic acid signal transduction. Plant Molecular Biology 80:519-537.

Elvira MI, Galdeano MM, Gilardi P, Garcialuque I, Serra M (2008). Proteomic analysis of pathogenesis-related proteins (PRs) induced by compatible and incompatible interactions of pepper mild mottle virus (PMMoV) in Capsicum chinense $L^{3}$ plants. Journal of Experimental Botany 59:1253-1265.

Fang LJ, Qin RL, Liu Z, Liu CR, Gai YP, Ji XL (2019). Expression and functional analysis of a PR-1 gene, MuPRl, involved in disease resistance response in mulberry (Morus multicaulis). Journal of Plant Interactions 14:376-385.

Farré EM, Harmer SL, Harmon FG, Yanovsky MJ, Kay SA (2005). Overlapping and distinct roles of PRR7 and PRR9 in the Arabidopsis circadian clock. Current Biology 15:47-54.

Farré EM, Kay SA (2007). PRR7 protein levels are regulated by light and the circadian clock in Arabidopsis. The Plant Journal 52:548-560.

Farré EM, Liu T (2013). The PRR family of transcriptional regulators reflects the complexity and evolution of plant circadian clocks. Current Opinion in Plant Biology 16:621-629.

Filichkin SA, Breton G, Priest HD, Dharmawardhana P, Jaiswal P, Fox SE, ... Mockler TC (2011). Global profiling of rice and poplar transcriptomes highlights key conserved circadian-controlled pathways and cis-regulatory modules. PLoS ONE 6:e16907.

Fukushima A, Kusano M, Nakamichi N, Kobayashi M, Hayashi N, Sakakibara H, Mizuno T, Saito K (2009). Impact of clockassociated Arabidopsis pseudo-response regulators in metabolic coordination. Proceedings of the National Academy of Sciences 106:7251-7256.

Garg A, Orru R, Ye W, Distler U, Chojnacki JE, Kohn M, ... Wolf E (2019). Structural and mechanistic insights into the interaction of the circadian transcription factor BMAL1 with the KIX domain of the CREB-binding protein. Journal of Biological Chemistry 294(45):16604-16619.

Greenham K, Mcclung CR (2015). Integrating circadian dynamics with physiological processes in plants. Nature Reviews Genetics 16:598-610.

He JD, Dong T, Wu HH, Zou YN, Wu QS, Kuča K (2019). Mycorrhizas induce diverse responses of root TIP aquaporin gene expression to drought stress in trifoliate orange. Scientia Horticulturae 243:64-69.

He JD, Zou YN, Wu QS, Kuča K (2020). Mycorrhizas enhance drought tolerance of trifoliate orange by enhancing activities and gene expression of antioxidant enzymes. Scientia Horticulturae 262:108745.

Jain N, Vergish S, Khurana JP (2018). Validation of house-keeping genes for normalization of gene expression data during diurnal/circadian studies in rice by RT-qPCR. Scientific Reports 8:3203.

Juliana MG, Aparecida RF, Renata FP, Claire B, Thiago JN, Brandon C, ... Alexandre N (2014). Diurnal oscillations of soybean circadian clock and drought responsive genes. PLoS ONE 9:e86402.

Khan S, Rowe SC, Harmon FG (2010). Coordination of the maize transcriptome by a conserved circadian clock. BMC Plant Biology 10:126.

Kim JA, Yang TJ, Kim JS, Park JY, Kwon SJ, Lim MH, Jin M, Lee SC, Lee SI, Choi BS, Um SH, Kim HI, Chun C, Park BS (2007). Isolation of circadian-associated genes in Brassica rapa by comparative genomics with Arabidopsis thaliana. Molecules and Cells 23:145-153.

Li J, Liu YH, Zhang Y, Chen C, Yu X, Yu SW (2017). Drought stress modulates diurnal oscillations of circadian clock and drought-responsive genes in Oryza sativa L. Hereditas 39:837-846.

Liu T, Carlsson J, Takeuchi T, Newton L, Farre EM (2013). Direct regulation of abiotic responses by the Arabidopsis circadian clock component PRR7. The Plant Journal 76:101-114. 
138

Livak KJ, Schmittgen TD (2001). Analysis of relative gene expression data using real-time quantitative PCR and the $2-{ }^{\Delta \Delta} \mathrm{CT}$ method. Methods 25:402-408.

Makino S, Kiba T, Imamura A, Hanaki N, Nakamura A, Suzuki T, ... Mizuno T (2000). Genes encoding pseudo-response regulators: insight into his-to-asp phosphorelay and circadian rhythm in Arabidopsis thaliana. Plant and Cell Physiology 41:791-803.

Michael TP, Mockler TC, Breton G, McEntee C, Byer AD, Trout JP, ... Chory J (2008). Network discovery pipeline elucidates conserved time-of-day-specific cis-regulatory modules. PLoS Genetics 4:e14.

Murakami M, Tago Y, Yamashino T, Mizuno T (2007). Comparative overviews of clock-associated genes of Arabidopsis thaliana and Oryza sativa. Plant and Cell Physiology 48:110-121.

Nakamichi N, Kiba T, Henriques R, Mizuno T, Chua NH, Sakakibara H (2010). pseudo-response regulators 9, 7, and 5 are transcriptional repressors in the Arabidopsis circadian clock. The Plant Cell 22:594-605.

Nakamichi N, Takao S, Kudo T, Kiba T, Wang Y, Kinoshita T, Sakakibara H (2016). Improvement of Arabidopsis biomass and cold, drought and salinity stress tolerance by modified circadian clock-associated pseudo-response regulators. Plant and Cell Physiology 57:1085-1097.

Nohales MA, Kay SA (2016). Molecular mechanisms at the core of the plant circadian oscillator. Nature Structural \& Molecular Biology 23:1061-1069.

Osakabe Y, Yamaguchi-Shinozaki K, Shinozaki K, Tran LP (2013). Sensing the environment: key roles of membrane-localized kinases in plant perception and response to abiotic stress. Journal of Experimental Botany 64:445-458.

Para A, Farre EM, Imaizumi T, Pruneda-Paz JL, Harmon FG, Kay SA (2007). PRR3 is a vascular regulator of TOC1 stability in the Arabidopsis circadian clock. The Plant Cell 19:3462-3473.

Poggi I, Polidori JJ, Gandoin JM, Paolacci V, Battini M, Albertini M, Ameglio T, Cochard H (2007). Stomatal regulation and xylem cavitation in Clementine (Citrus clementina Hort) under drought conditions. Journal of Horticultural Science \& Biotechnology 82:845-848.

Riviere MP, Marais A, Ponchet M, Willats W, Galiana E (2008). Silencing of acidic pathogenesis-related PR- 1 genes increases extracellular $\beta$ - $(1 \rightarrow 3)$-glucanase activity at the onset of tobacco defence reactions. Journal of Experimental Botany 59:1225-1239.

Saitou N, Nei M (1987). The neighbor-joining method: a new method for reconstructing phylogenetic trees. Molecular Biology and Evolution 4:406-425.

Santana-Vieira DDS, Freschi L, Almeida LADH, Moraes DHSD, Neves DM, Santos LMD, ... Gesteira ADS (2016). Survival strategies of citrus rootstocks subjected to drought. Scientific Reports 6:38775.

Seo PJ, Mas P (2015). Stressing the role of the plant circadian clock. Trends in Plant Science 20:230-237.

Sitnikova T, Rzhetsky A, Nei M (1995). Interior-branch and bootstrap tests of phylogenetic trees. Molecular Biology and Evolution 12:319-333.

Syed NH, Prince SJ, Mutava RN, Patil G, Li S, Chen W, ... Nguyen HT (2015). Core clock, SUB1, and ABAR genes mediate flooding and drought responses via alternative splicing in soybean. Journal of Experimental Botany 66: 1-21.

Takata N, Saito S, Saito CT, Uemura M (2010). Phylogenetic footprint of the plant clock system in angiosperms: evolutionary processes of pseudo-response regulators. BMC Evolutionary Biology 10:126.

Wang J, Zhang L, Cao Y, Qi C, Li S, Liu L, ... Guo YD (2018). CsATAF1 positively regulates drought stress tolerance by an ABA-dependent pathway and by promoting ROS scavenging in cucumber. Plant and Cell Physiology 59:930-945.

Wang L, Kim J, Somers DE (2013). Transcriptional corepressor TOPLESS complexes with pseudo response regulator proteins and histone deacetylases to regulate circadian transcription. Proceedings of the National Academy of Sciences 110:761766.

Wu QS, He JD, Srivastava AK, Zou YN, Kuca K (2019). Mycorrhizas enhance drought tolerance of citrus by altering root fatty acid compositions and their saturation levels. Tree Physiology 39:1149-1158.

Wu QS, Srivastava AK, Zou YN (2013). AMF-induced tolerance to drought stress in citrus: a review. Scientia Horticulturae 164:77-87.

Yerushalmi S, Green RM (2009). Evidence for the adaptive significance of circadian rhythms. Ecology Letters 12:970-981.

Zhang F, Zou YN, Wu QS, Kuča K (2020). Arbuscular mycorrhizas modulate root polyamine metabolism to enhance drought tolerance of trifoliate orange. Environmental and Experimental Botany 171:103962. 
The journal offers free, immediate, and unrestricted access to peer-reviewed research and scholarly work. Users are allowed to read, download, copy, distribute, print, search, or link to the full texts of the articles, or use them for any other lawful purpose, without asking prior permission from the publisher or the author.

OPEN ACCESS

License - Articles published in Notulae Botanicae Horti Agrobotanici Cluj-Napoca are Open-Access, distributed under the terms and conditions of the Creative Commons Attribution (CC BY 4.0) License.

(C) Articles by the authors; UASVM, Cluj-Napoca, Romania. The journal allows the author(s) to hold the copyright/to retain publishing rights without restriction. 